\title{
Serum calcium improved systemic inflammation marker for predicting survival outcome in rectal cancer
}

\author{
Zhuokai Zhuang $^{1,2 \#}$, Xiaolin Wang ${ }^{1 \#}$, Meijin Huang ${ }^{1,2}$, Yanxin Luo ${ }^{2}$, Huichuan Yu ${ }^{1}$ \\ ${ }^{1}$ Guangdong Institute of Gastroenterology, Guangdong Provincial Key Laboratory of Colorectal and Pelvic Floor Disease, The Sixth Affiliated \\ Hospital, Sun Yat-sen University, Guangzhou, China; ${ }^{2}$ Department of Colorectal Surgery, The Sixth Affiliated Hospital, Sun Yat-sen University, \\ Guangzhou, China \\ Contributions: (I) Conception and design: H Yu; (II) Administrative support: M Huang; (III) Provision of study materials or patients: M Huang, \\ Y Luo; (IV) Collection and assembly of data: H Yu, Z Zhuang, X Wang; (V) Data analysis and interpretation: H Yu, Z Zhuang, X Wang; (VI) \\ Manuscript writing: All authors; (VII) Final approval of manuscript: All authors. \\ \#These authors contributed equally to this work. \\ Correspondence to: Yanxin Luo, MD, PhD. Department of Colorectal Surgery, The Sixth Affiliated Hospital, Sun Yat-sen University, 26 Yuancun \\ Erheng Road, Guangzhou 510655, China. Email: luoyx25@mail.sysu.edu.cn; Huichuan Yu, MD, PhD. Guangdong Institute of Gastroenterology, \\ Guangdong Provincial Key Laboratory of Colorectal and Pelvic Floor Disease, The Sixth Affiliated Hospital, Sun Yat-sen University, 26 Yuancun \\ Erheng Road, Guangzhou 510655, China. Email: yuhch5@mail.sysu.edu.cn.
}

Background: Systemic inflammation markers have shown prognostic values with variability in rectal cancer. Considering the association of serum calcium with inflammation, we aimed to examine whether it could improve systemic inflammation markers for survival prediction.

Methods: We enrolled 508 patients with stage I to III rectal cancer who underwent curative resection. The cohort was grouped by corrected serum calcium (cCa), platelet-to-lymphocyte ratio (PLR), and CaPLR (a score model combining cCa with PLR) for survival analysis. The LR (likelihood ratio) test and AIC (Akaike information criterion) were applied to compare models in survival prediction. The primary endpoint was disease-free survival (DFS).

Results: A total of $26.7 \%(136 / 508)$ patients reached recurrence after curative surgery. Both high cCa (HR 1.486; 95\% CI, 1.018-2.171; P=0.040) and high PLR (HR 1.452; 95\% CI, 1.059-1.991; P=0.021) were significantly associated with worse DFS. In model comparison, the AIC and LR were improved after cCa was added to PLR model in DFS prediction (AIC: 1,704.83 vs. 1,707.14 vs. 1,707.15; LR: 8.68 vs. 4.37 vs. 4.36 ; $\mathrm{P}=0.037$ ). The CaPLR was developed for DFS prediction with adjusted HRs of 2.216 (95\% CI, 1.256-3.909; $\mathrm{P}=0.006)$ and 1.679 (95\% CI, 1.004-2.836; $\mathrm{P}=0.047)$ for high and intermediate score group respectively compared to low score group. A nomogram for predicting DFS was generated by using CaPLR and other clinical predictors, with a concordance index of 0.705 (95\% CI, 0.620-0.789; $\mathrm{P}<0.001$ ).

Conclusions: Serum calcium could improve systemic inflammation markers in survival prediction for patients with rectal cancer.

Keywords: Serum calcium; platelet-to-lymphocyte ratio (PLR); systemic inflammation; rectal cancer

Submitted Oct 31, 2020. Accepted for publication Jan 18, 2021.

doi: 10.21037/jgo-20-479

View this article at: http://dx.doi.org/10.21037/jgo-20-479

\section{Introduction}

Colorectal cancer (CRC) is known as the third common cancer and contributes the second most to cancer death $(1,2)$. The current standard treatment for non-metastatic rectal cancer is total mesorectal excision (TME) or combination treatments, such as surgery with chemo/radiotherapy (3). However, the survival outcomes after surgery vary in different patients, which makes it essential to stratify patients by 
different risk of recurrence and death to avoid overtreatment or insufficient therapy. The period immediately around surgery for tumors has been supposed to be a critical time that facilitates tumor growth and metastasis $(4,5)$. Therefore, great efforts have been made to develop predictive markers for long-term outcomes by using clinicopathological variables collected from perioperative care in rectal cancer patients.

It has been suggested that inflammation plays a vital role in tumorigenesis and development of CRC $(6,7)$. In this context, some models based on markers representing the systemic inflammation status in perioperative period, such as platelet-to-lymphocyte ratio (PLR), have been proposed to predict prognosis of CRC patients in multiple studies (8-10). However, their prognostic values remain controversial with high variability and limited reproducibility $(10,11)$, suggesting a dramatic heterogeneity among cancer populations, and the current inflammation marker panels need to be improved by combining other markers.

Calcium signaling has been recognized as a crucial role in tumor immune regulation and inflammatory promotion. The emerging data have shown that not only changes of calcium ions level can remodel channels in inflammation cells which play an important role in the perioperative immune system, but alterations of calcium levels are associated with several tumor-related proinflammatory cytokines (12-14). There has been evidence from cohort studies suggesting an association of elevated serum calcium with low-level chronic inflammation in populations (15). Previous studies have shown the prognostic value of serum calcium in multiple cancers, including prostate, breast, and lung cancers (16-19). Furthermore, a study demonstrated that serum calcium could contribute to an inflammationbased modified Glasgow Prognostic Score (mGPS) model for survival prediction in multiple cancers (20).

On the basis of the aforementioned association of serum calcium with inflammation and cancer progression, we aimed to explore the prognostic value of serum calcium and investigate whether the model that combines serum calcium and systemic inflammation markers could improve survival prediction. We speculated that this comprehensive inflammation panel might achieve a considerable improvement in predicting prognosis for patients with rectal cancer. We present the following article in accordance with the TRIPOD reporting checklist (available at http:// dx.doi.org/10.21037/jgo-20-479).

\section{Methods}

\section{Patient coborts}

We retrospectively studied the patients that were prospectively and consecutively enrolled in the institutional database program of colorectal disease (IDPCD) as previously described $(21,22)$. Patients were included if they were pathologically diagnosed with stage I to III rectal cancer. The patients with familial adenomatous polyposis (FAP), multiple primary cancers, inflammatory bowel disease, hepatic or renal insufficiency, end-stage lung diseases and thyroid disease were excluded. Rectal cancer was staged according to the 7 th edition of the American Joint Committee on Cancer (AJCC) staging system (23). Staging procedures included contrast-enhanced computed tomography (CT) scans of the thorax, abdomen, and pelvis, pelvic MRIs and colonoscopy.

\section{Measurement of inflammation-associated markers}

Serum calcium was measured photometrically by the clinical laboratory in our institute, on a PRISMA multichannel autoanalyzer (Clinicon AB, Bromma, Sweden). According to the IDPCD protocols in our institution, the enrolled patients were consented to test preoperative serum calcium levels and blood cell counts within the 24 hours after admission to the hospital. Since total calcium changes with albumin concentration, it was corrected by serum albumin level to obtain the corrected serum calcium $(\mathrm{cCa}): \mathrm{cCa}=$ total calcium $+[0.8 \times(4.0-$ serum albumin $)](24)$. PLR was defined as the absolute platelet count divided by the absolute lymphocyte count, and a cut-off value of 150 was adopted to discriminate patients with low versus high PLR as previously described (25-27).

\section{Treatment, follow-up and study endpoints}

Patients were treated and followed up according to NCCN guidelines-based protocols in our institute $(28,29)$. Briefly, all patients underwent TME, and $13.1 \%$ patients received neoadjuvant chemo/radiotherapy. Surgical approaches included low anterior resections, abdominoperineal resections, and Parks procedure. Postoperative assessment of TME quality was performed in all surgical specimens. According to physicians' suggestions and patients' decisions, 5-fluorouracilbased chemotherapy was given after operation. Patients were 
followed up every three months for the first two years and every six months thereafter. Each visit consisted of pertinent medical history, physical examination, including rectal examination, and measurement of serum carcinoembryonic antigen (CEA) levels. Colonoscopy and radiological examinations consisting of chest radiography, abdominopelvic $\mathrm{CT}$ and ultrasonography were scheduled every six months for the first three years and annually thereafter. Cancer recurrence was detected by CEA $>5 \mathrm{ng} / \mathrm{mL}$ and/or a sequential computerized tomography scan with evidence of the disease followed by histopathological confirmation. The primary endpoint was disease-free survival (DFS), which was defined as the time from the surgery until recurrence or death from any cause.

\section{Statistical analyses}

The intergroup comparisons of clinicopathologic variables were performed using the analysis of variance and KruskalWallis tests for continuous variables depending on their distributions, and the chi-square and two-tailed Fisher's exact tests were used for categorical variables. According to the optimum cutoff point determined by maximally selected rank statistics, the cohort was divided into high and low cCa group. The Cox proportional hazard model was applied to generate the CaPLR score. According to CaPLR score, patients were divided into three groups, including high score group (HS, score $=0.745$ ), intermediate score group (IS, score $=0.397$ or 0.348 ), and low score group $(\mathrm{LS}$, score $=0)$. The Kaplan-Meier method was used to estimate the long-term survival. A univariate screen of potential predictors of mortality by Cox proportional hazard model for each candidate variable was performed, and the selected predictors with statistical significance were included in the multivariate Cox model to examine their independently predictive value. The comparison between predictive models was assessed using likelihood ratio (LR) and Akaike information criterion (AIC), as previously described $(30,31)$, in competing models including or not including $\mathrm{cCa}$ or CaPLR score. In general, the model with a lower AIC and a higher LR were considered a better one. The predictive nomogram was developed based on the results of multivariate Cox regression analysis. The Harrell's concordance index (C-index) of the nomogram was calculated using the bootstrap method to evaluate the discriminative ability of the nomogram. Data analyses were performed using SPSS version 22.0 for Windows (SPSS, Inc., Chicago, IL) and R version 3.5.1 (R-project, Institute for Statistics and Mathematics, Vienna, Austria). A two-side $\mathrm{P}$ value $<0.05$ was considered statistically significant.

\section{Ethics statement}

The study was conducted in accordance with the Declaration of Helsinki (as revised in 2013). This study was approved by the Institutional Review Board of the Sixth Affiliated Hospital of Sun Yat-sen University (No. 2017ZSLYEC-006) and informed consent was taken from all the patients.

\section{Results}

\section{Baseline characteristics}

Between June 2007 and June 2011, a total of 795 patients with pathologically confirmed rectal cancer received curative surgery. In this cohort, 508 patients matched the inclusion and exclusion criteria and were included (Figure 1). Among them, 26.7\% (136/508) patients reached recurrence after curative surgery. The demographics and clinicopathological features were shown in Table 1. There were 296 male and 212 female patients, with a median age of 59 years (21-89 years). The AJCC staging among patients was distributed as $26.2 \%, 31.3 \%$ and $42.5 \%$ for stages I, II and III, respectively. Patients were grouped by preoperative $\mathrm{cCa}$ based on the optimal cutoff value determined by maximally selected rank statistics (Figure 2A). The low and high cCa group had 137 and 371 patients respectively. There was no significant difference in baseline characteristics between two groups (Table 1).

\section{Predicting value of serum calcium for survival outcome in rectal cancer}

We performed a screen for prognostic markers by using univariate Cox analysis, in which the high $\mathrm{cCa}$ was found to be significantly associated with worse DFS ( $\mathrm{HR}=1.486$, 95\% CI, 1.018-2.171; P=0.040; Table 2). In the KaplanMeier curve, significantly worse survival was observed in patients with high $\mathrm{cCa}$ compared to those with low $\mathrm{cCa}$ $(\mathrm{P}=0.038$; Figure $2 B)$. In addition, high PLR, representing a high systemic inflammation status, was also found to predict worse DFS in this cohort (HR $=1.452,95 \%$ CI, 1.059-1.991; $\mathrm{P}=0.021$; Figure $2 C$, Table 2). The other markers that were significantly associated with DFS in the univariate Cox analysis included age, TNM stage, differentiation degree, 


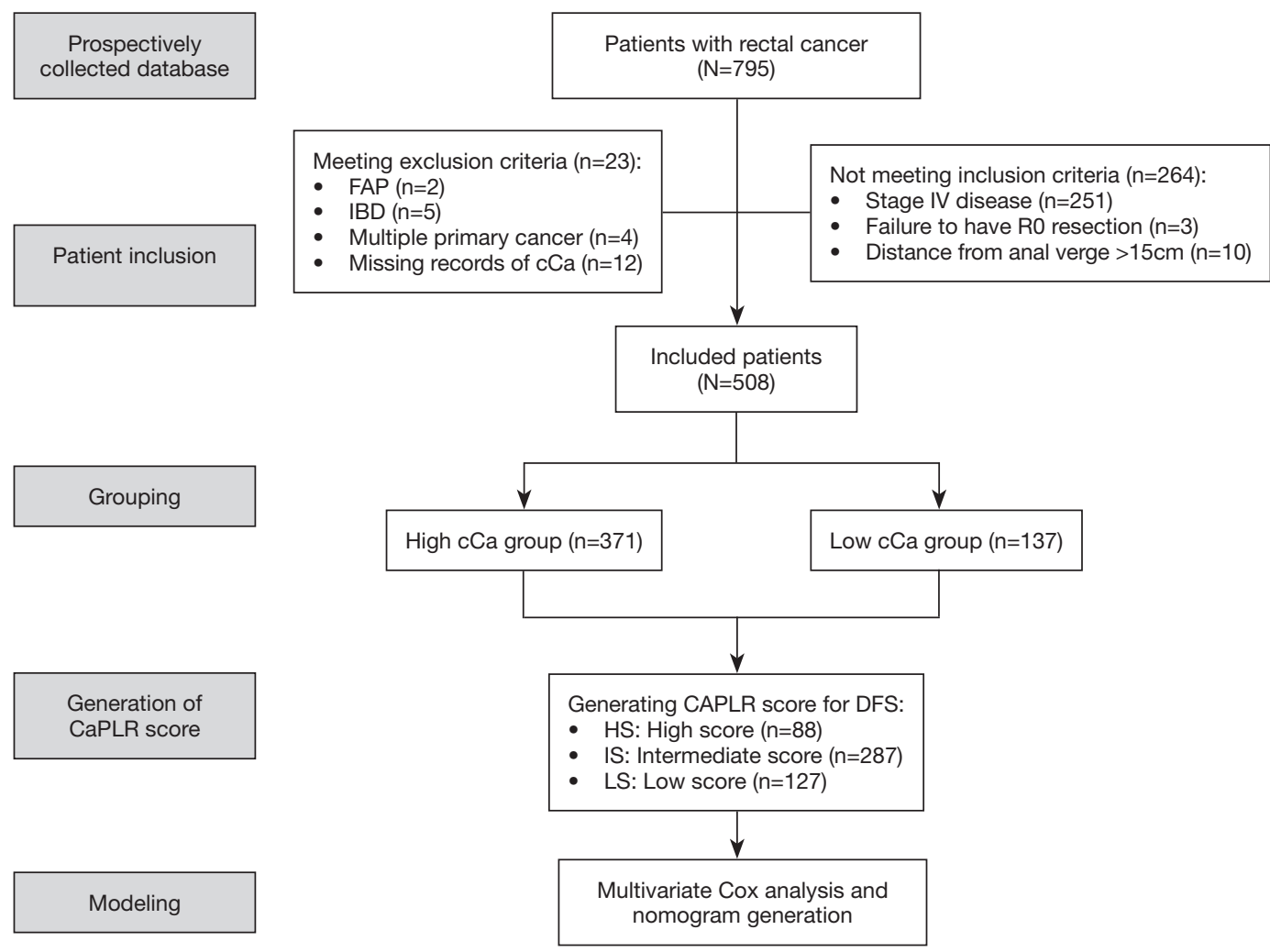

Figure 1 Patient disposition in the analysis of the association between serum calcium and long-term outcomes in rectal cancer. FAP, familial adenomatous polyposis; IBD, inflammatory bowel disease; cCa, corrected serum calcium; DFS, disease-free survival.

lymphovascular invasion, perineural invasion, preoperative CEA, and distance from anal verge (Table 2).

\section{Serum calcium adds values to current prognostic makers}

Considering the aforementioned association of serum calcium with inflammation, we applied LR test and AIC to explore how cCa could improve current systemic inflammation marker in predicting survival outcome. As shown in Table 3, the model 3 including both PLR and $\mathrm{cCa}$ had a lower AIC and a higher LR in comparison to the model 1 or model 2 that only included PLR or cCa (AIC: $1,704.83$ vs. $1,707.14$ vs. 1,707.15; LR: 8.68 vs. 4.37 vs. 4.36; $\mathrm{P}=0.037$; Table 3). This result showed that the model combined PLR and cCa may perform better in DFS prediction. We therefore constructed a CaPLR score model for further survival analysis by using Cox proportional hazard model with their grouping information (1 for high cCa or PLR group, and 0 for low cCa or PLR group) and coefficients in the regression: $\mathrm{CaPLR}$ score $=\mathrm{cCa} \times 0.348+$ PLR $\times 0.397$.

\section{CaPLR model improved survival prediction in rectal cancer}

Patients were categorized into three groups based on CaPLR. There were 88 patients in the HS group, 287 in the IS group, and 127 in the LS group. In the KaplanMeier analysis, the three groups had a significant difference in survival curve, in which the patients with higher CaPLR had significantly worse survival $(\mathrm{P}=0.005$, Figure $2 D)$. The HS and IS group had HRs of 2.418 (95\% CI, 1.390-4.206; $\mathrm{P}=0.002)$ and 1.892 (95\% CI, 1.128-3.175; $\mathrm{P}=0.016$ ) respectively in comparison to LS group in Cox analysis (Table 2).

We further performed outcome analysis between patients grouped by CaPLR score. The total recurrence rate was $23.3 \%(50 / 215)$ in the whole cohort, and HS group had $18.2 \%$ higher risk of recurrence compared with LS group [30.7\% (39/127) vs. $12.5 \%$ (11/88), $\mathrm{P}=0.002]$ (Table 4). In addition, both the local recurrence $[4.6 \%(4 / 88)$ vs. $13.6 \%$ (17/127), $\mathrm{P}=0.031]$ and distant metastasis [9.2\% (8/88) vs. $25.6 \%(32 / 127), \mathrm{P}=0.003]$ rate were significantly higher in 
Table 1 Baseline characteristics between patients with high and low level of preoperative serum calcium

\begin{tabular}{|c|c|c|c|c|}
\hline Variables & Total $(\mathrm{N}=508)$ & Low cCa (N=137) & High cCa $(\mathrm{N}=371)$ & $P$ value \\
\hline BMI, median [range] & $22.1[13.3-35.9]$ & $22.3[15.8-33.8]$ & $22.1[13.3-35.9]$ & 0.643 \\
\hline Sex, n (\%) & & & & 0.211 \\
\hline Male & $296(58.3)$ & $86(62.8)$ & $210(56.6)$ & \\
\hline TNM stage, AJCC, n (\%) & & & & 0.056 \\
\hline I & $133(26.2)$ & $28(20.4)$ & $105(28.3)$ & \\
\hline II & $159(31.3)$ & $53(38.7)$ & $106(28.6)$ & \\
\hline III & $216(42.5)$ & $56(40.9)$ & $160(43.1)$ & \\
\hline Moderate-high & $396(80.8)$ & $110(81.5)$ & $286(80.6)$ & \\
\hline Lymphovascular invasion, $\mathrm{n}(\%)$ & & & & 0.962 \\
\hline Negative & $464(91.3)$ & $125(91.2)$ & $339(91.4)$ & \\
\hline Positive & $44(8.7)$ & $12(8.8)$ & $32(8.6)$ & \\
\hline Perineural invasion, n (\%) & & & & 0.678 \\
\hline Negative & $457(90.0)$ & $122(89.1)$ & $335(90.3)$ & \\
\hline Positive & $51(10.0)$ & $15(10.9)$ & $36(9.7)$ & \\
\hline Preoperative CEA, n (\%) & & & & 0.094 \\
\hline Preoperative PLR, n (\%) & & & & 0.901 \\
\hline Low & $327(65.1)$ & $88(64.7)$ & $239(65.3)$ & \\
\hline High & $175(34.9)$ & $48(35.3)$ & $127(34.7)$ & \\
\hline Blood transfusion, $\mathrm{n}(\%)$ & & & & 0.750 \\
\hline No & $422(83.1)$ & $115(83.9)$ & 307 (82.7) & \\
\hline Yes & $86(16.9)$ & $22(16.1)$ & $64(17.3)$ & \\
\hline Neoadjuvant treatment, n (\%) & & & & 0.090 \\
\hline No & $438(86.9)$ & $123(91.1)$ & $315(85.4)$ & \\
\hline Yes & $66(13.1)$ & $12(8.9)$ & $54(14.6)$ & \\
\hline Adjuvant treatment, n (\%) & & & & 0.664 \\
\hline No & 235 (46.9) & $65(48.5)$ & $170(46.3)$ & \\
\hline Yes & $266(53.1)$ & $69(51.5)$ & $197(53.7)$ & \\
\hline
\end{tabular}

cCa, corrected serum calcium; BMI, body mass index; CEA, carcinoembryonic antigen; PLR, platelet-to-lymphocyte ratio.

(C) Journal of Gastrointestinal Oncology. All rights reserved. 
A$$
\text { A }
$$
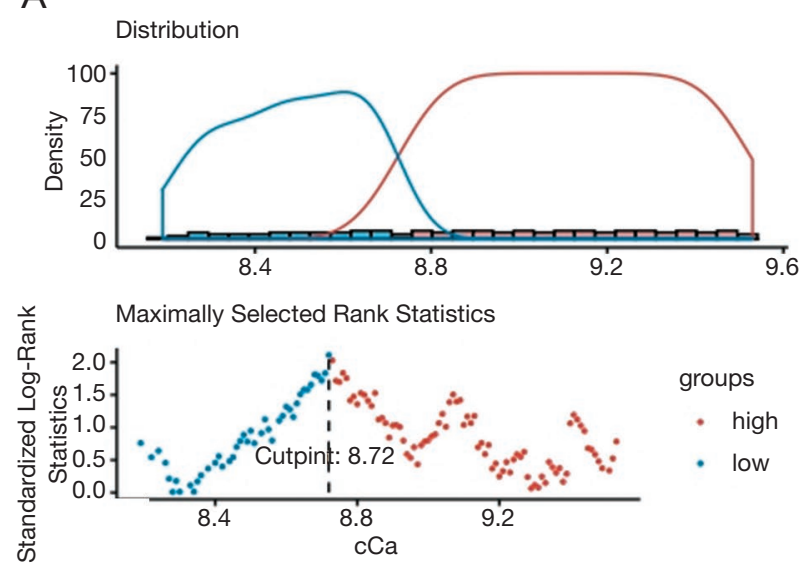
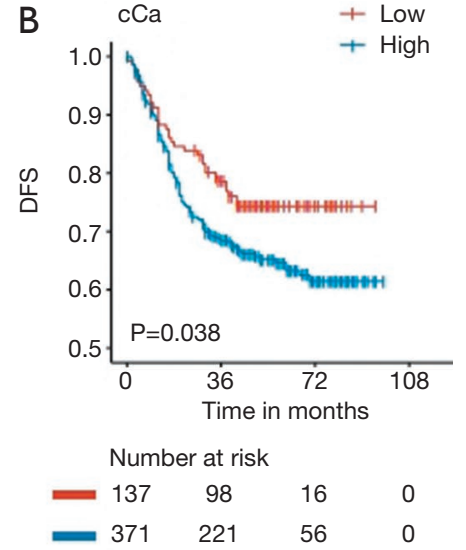

C
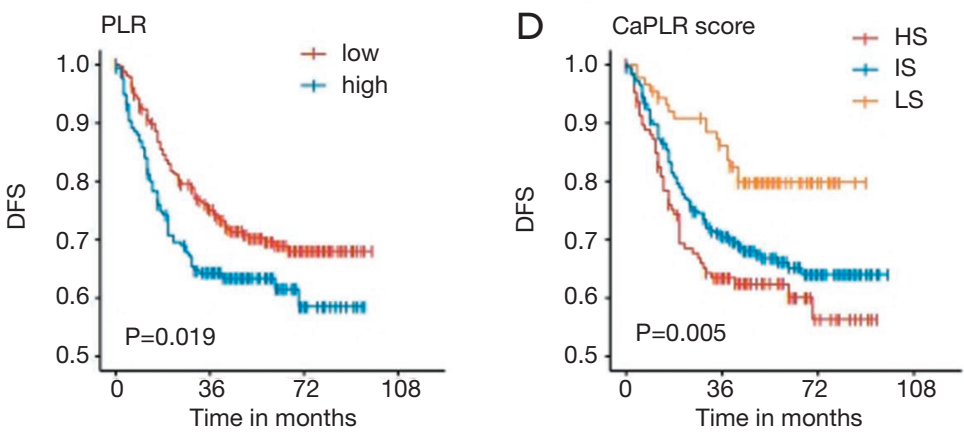

\begin{tabular}{cccc}
\multicolumn{3}{l}{ Number at risk } & \\
332 & 225 & 52 & 0 \\
117 & 95 & 18 & 0
\end{tabular}

\begin{tabular}{|c|c|c|c|c|}
\hline \multicolumn{4}{|c|}{ Number at risk } & \\
\hline 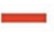 & 127 & 67 & 14 & 0 \\
\hline & 287 & 178 & 43 & \\
\hline$=$ & 88 & 71 & 12 & 0 \\
\hline
\end{tabular}

Figure 2 Survival analysis of predictive variables in patients with rectal cancer. (A) The cutoff point of cCa was determined by using maximally selected rank statistics. Kaplan-Meier curves showed the difference in disease-free survival (DFS) between patients grouped by preoperative corrected serum calcium (B) and platelet-to-lymphocyte ratio (C). Patients were divided into three groups based on CaPLR score (D), including high score group (HS, score $=0.745$ ), intermediate score group (IS, score $=0.397$ or 0.348), and low score group (LS, score $=0$ ). The survival curves showed the difference in DFS among the groups. The log-rank test was applied and $\mathrm{P}$ value was given in each plot. cCa, corrected serum calcium.

HS group (Table 4).

In the multivariate Cox analysis including CaPLR score and other prognostic predictors from univariate screen, the CaPLR score was further proved to be an independent predictor for DFS with adjusted HRs of 2.216 (95\% CI, 1.256-3.909; $\mathrm{P}=0.006)$ and 1.679 (95\% CI, 1.004-2.836; $\mathrm{P}=0.047)$ for HS and IS group respectively in comparison to LS group (Table 2). Moreover, the TNM stage, lymphovascular invasion, CEA and distance from anal verge were also independent predictors in the multivariate Cox model (Table 2).

Next, we applied LR test and AIC to explore how CaPLR could add prognostic values to current predictive panels in rectal cancer. In the comparison between models 4 and 5, after CaPLR was added to TNM stage, a dramatic improvement was observed in AIC and LR (AIC: 1,666.57 vs. 1,659.94; LR: 44.95 vs. 53.57, $\mathrm{P}=0.003$; Table 3). A similar improvement was found in the comparison between models 6 and 7, in which the multivariate Cox model was improved after CaPLR was added (AIC: 1,656.56 vs. 1,650.21; LR: 60.96 vs. 69.31, $\mathrm{P}=0.003$; Table 3). Thus, both the widelyused AJCC TNM staging panel and the generated Cox model in this study may get increased discriminatory ability for predicting survival outcome with CaPLR score.

We also compared the short-term outcomes between patients stratified by CaPLR score. Intriguingly, in the 
Table 2 Univariate and multivariate Cox analysis of predictive variables for disease-free survival

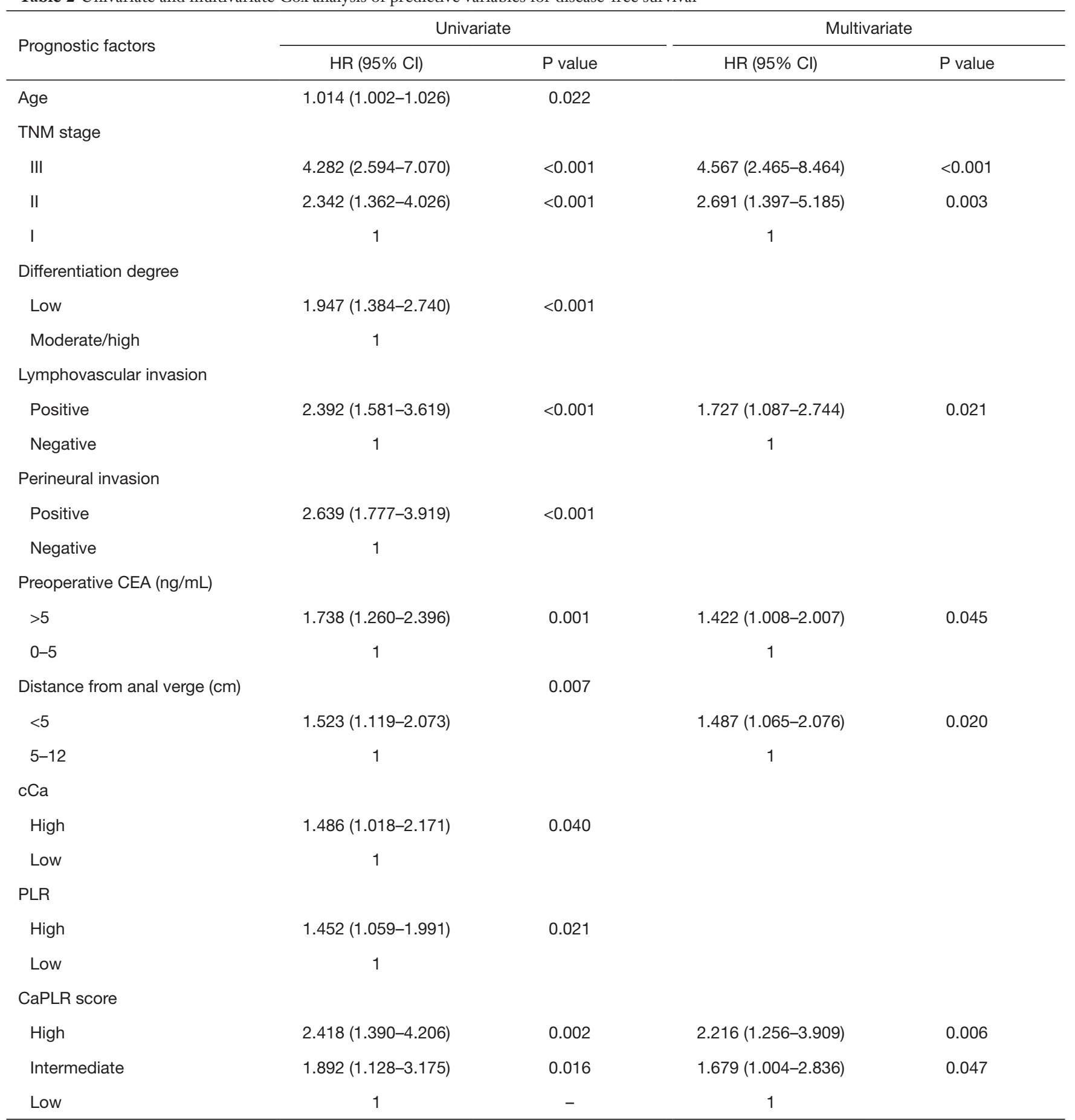

The Cox survival analysis has been applied to all clinicopathological variables, and only significant results were shown in this table. HR, hazard ratio; Cl, confidential interval; DFS, disease-free survival; CEA, carcinoembryonic antigen; PLR, platelet-to-lymphocyte ratio; cCa, corrected serum calcium; CaPLR, cCa-PLR panel score. 
Table 3 Model fit among seven models including or not including preoperative cCa

\begin{tabular}{lcccc}
\hline Models & Df & AIC & LR & P value \\
\hline Model 1 & 1 & $1,707.14$ & 4.37 & $0.037^{\mathrm{a}}$ \\
Model 2 & 1 & $1,707.15$ & 4.36 & $0.037^{\mathrm{b}}$ \\
Model 3 & 2 & $1,704.83$ & 8.68 & 44.95 \\
Model 4 & 1 & $1,666.57$ & 53.57 & $0.003^{\mathrm{c}}$ \\
Model 5 & 2 & $1,659.94$ & 60.96 & $0.003^{\mathrm{d}}$ \\
Model 6 & 4 & $1,656.56$ & 69.31 & \\
Model 7 & 5 & $1,650.21$ & & \\
\hline
\end{tabular}

Model 1 includes PLR. Model 2 includes cCa. Model 3 includes PLR and cCa. Model 4 includes TNM stage. Model 5 includes TNM stage, and CaPLR score. Model 6 includes TNM stage, differentiation degree, lymphovascular invasion, perineural invasion, preoperative CEA, and distance from anal verge. Model 7 includes CaPLR score and all variables in Model 6. ${ }^{\mathrm{a}, \mathrm{b}}$, P values for the LR test in model 1 and 2 compared with model 3; ${ }^{\mathrm{c}, \mathrm{d}}, \mathrm{P}$ values for the LR test in model 5 and 6 compared with the model above. Df, degrees of freedom; AIC, Akaike information criterion value; LR, likelihood ratio; CEA, carcinoembryonic antigen.

association analysis between CaPLR and complication incidence after surgery, high CaPLR score was significantly related to increased risk of anastomotic stenosis $[14.8 \%$ (13/88) vs. 5.5\% (7/127), $\mathrm{P}=0.022$, Table 4].

\section{A nomogram for prognosis evaluation in rectal cancer}

A nomogram for predicting DFS outcome was generated using the variables from the multivariate Cox model, including CaPLR score, TNM stage, lymphovascular invasion, CEA, and distance from anal verge (Figure 3). The calibration curves for the nomogram were shown. The C-index of the nomogram for predicting DFS was 0.705 (95\% CI, 0.620-0.789; $\mathrm{P}<0.001$ ).

\section{Discussion}

The main finding of this work is that a novel inflammationbased prognostic panel that combined PLR with cCa had significantly improved value for predicting survival outcome in rectal cancer. This improved inflammation marker panel that was first proposed in this study is supported by the analysis using prospective data from institutional database and medical records. In current study, we demonstrated that both high cCa and high PLR were significantly associated with worse survival outcomes. In model comparison by using AIC and LR test, cCa could significantly improve the performance of PLR in survival prediction. Based on this result, the CaPLR score was generated to stratify patients into three groups with sequential risk of recurrence and death, in which patients with higher CaPLR had significantly worse DFS. Furthermore, a nomogram that could well predict survival outcome was generated by using CaPLR score, TNM stage, lymphovascular invasion, CEA and distance from anal verge.

It has been well documented that systemic inflammation plays an important role in CRC tumorigenesis, proliferation, invasion, metastasis, angiogenesis, and suppressing antitumor immunity (6). Several systemic inflammation-based markers, such as PLR and mGPS, have been developed and found to have independent value in predicting cancer survival outcomes (11). An elevated preoperative PLR is usually accompanied by thrombocytosis or lymphocytopenia, implying an activated inflammatory status and repressed immune response of patients. Expectedly, PLR, a marker that represents the balance between two inflammatory status, has shown prognostic values in multiple studies (8-10). In addition, elevated platelets tend to gather tumor cells by release biological factors, help stimulate the development of new vessels by interacting with PDGF, VEGF and PF4, and activate DNA-damagepromoting agents, which may promote carcinogenesis (32).

The underlying mechanism of the association between serum calcium and inflammation includes the interaction with the extracellular calcium sensing receptor that has been reported to be a crucial regulator for inflammation through multiple downstream signaling pathways (33). In addition, the TRPV1 channel, involved in calcium signaling fundamental for many cellular processes, was recently documented to be expressed on the immune cells and activated by proinflammatory cytokines, and it was also reported to be aberrantly expressed on cancer cells (12). 
Table 4 Short-term and long-term outcomes after curative resection in patients with high and low CaPLR scores

\begin{tabular}{|c|c|c|c|c|}
\hline Clinical outcomes & Overall population (N=215), n (\%) & \multicolumn{2}{|c|}{ CaPLR score } & $P$ value \\
\hline Anastomotic complication ${ }^{1}$ & & & & 0.315 \\
\hline No & $180(83.7)$ & $71(80.7)$ & $109(85.8)$ & \\
\hline Yes & 35 (16.3) & $17(19.3)$ & $18(14.2)$ & \\
\hline No & $198(92.1)$ & 80 (90.9) & $118(92.9)$ & \\
\hline Yes & $17(7.9)$ & $8(9.1)$ & $9(7.1)$ & \\
\hline Anastomotic stenosis & & & & $0.022^{*}$ \\
\hline No & $195(90.7)$ & $75(85.2)$ & $120(94.5)$ & \\
\hline Alive & $174(80.9)$ & $75(85.2)$ & $99(78.0)$ & \\
\hline Death & $41(19.1)$ & $13(14.8)$ & $28(22.0)$ & \\
\hline Total recurrence ${ }^{2}$ & & & & $0.002^{*}$ \\
\hline No & $165(76.7)$ & $77(87.5)$ & $88(69.3)$ & \\
\hline Yes & $50(23.3)$ & $11(12.5)$ & $39(30.7)$ & \\
\hline Local recurrence & & & & $0.031^{*}$ \\
\hline No & $191(90.1)$ & $83(95.4)$ & $108(86.4)$ & \\
\hline Yes & $21(9.9)$ & $4(4.6)$ & $17(13.6)$ & \\
\hline
\end{tabular}

*, statistically significant $\mathrm{P}$ value. ${ }^{1}$, anastomotic complication refers to anastomotic leakage, bleeding or stenosis after operation in this study. ${ }^{2}$, total recurrence refers to patients with local recurrence and/or distant metastasis during follow-up after operation. CaPLR, cCaPLR panel score.

On the other side of the association with inflammation, calcium may promote cancer in other ways. First, calcium has been shown to be involved in cell differentiation and proliferation through modulating cell signaling that can commonly initiate colorectal carcinogenesis, such as APC/ $\beta$-catenin and Hedgehog pathways (34-37), and it has been proposed that hypercalcemic environment may help colon adenocarcinoma cells develop (38). Second, patients with bone metastasis are well known to be hypercalcemic (39) suggesting that patients with elevated serum calcium and worse survival might have undiagnosed metastatic disease before the curative-intent surgery. Moreover, hypercalcemia secondary to paraneoplastic lesions may contribute to the association between serum calcium and cancer survival. However, this condition frequently occurs in other cancers such as squamous cell lung cancer but rare in rectal cancer (40).

Although impressive prognostic values were found in several systemic inflammation-based markers, including PLR, mGPS, neutrophil-lymphocyte ratio, and Lymphocyteto-monocyte ratio, their predictive abilities varied and remained controversial among published cohorts $(10,11)$. We therefore developed additional markers to enable accurate identification of patients with high risk of recurrence and death by using inflammation-based panels. Our results showed that serum calcium could significantly improve the 

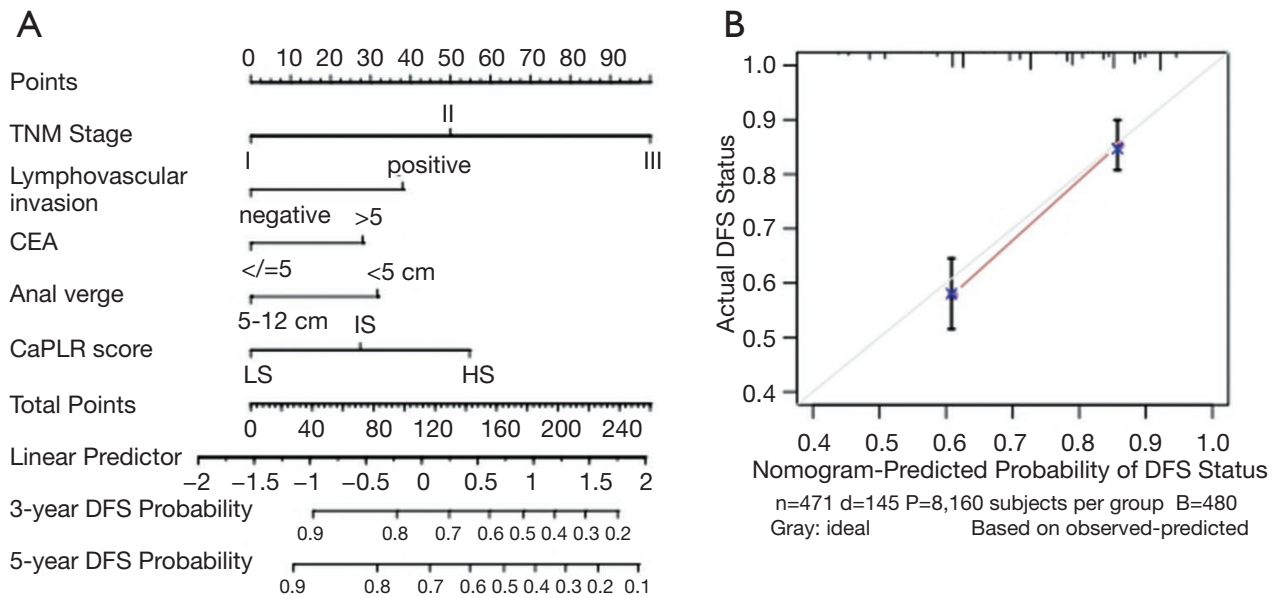

Figure 3 A nomogram and calibration curve for predicting DFS in CRC patients. (A) A nomogram to predict individual patient-level DFS based on CaPLR score and other clinicopathological risk factors. (B) Calibration plots for the validation sample of the nomogram. The average nomogram-predicted probability of DFS was plotted against observed DFS estimated by Kaplan-Meier. 95\% confidence intervals of the Kaplan-Meier estimates are indicated with vertical lines. The gray line indicates the reference line, showing where an ideal nomogram would lie. CEA, carcinoembryonic antigen; DFS, disease-free survival; CRC, colorectal cancer.

performance of systemic inflammation markers in survival prediction. After serum calcium was added, the CaPLR was generated and showed dramatically improved discriminability for survival outcomes. We further combined this improved inflammation-based marker with other known independent predictors to develop a nomogram that could well predict prognosis in a more comprehensive manner.

Nevertheless, limitations to our study exist. First, the results from retrospective analysis were limited by unknown confounders due to its observational nature, although multiple variables were controlled. Then, the total serum calcium varies with changes in albumin concentration and ionized calcium may be a more reliable marker, although the cCa, albumin-adjusted calcium, was analyzed instead. Since ionized calcium is not routinely measured in daily clinical practice, the value of serum calcium needs to be validated in a study that can prospectively test bloodderived ionized calcium.

\section{Conclusions}

Our results provided evidence that serum calcium could improve the value of systemic inflammation markers in survival prediction for patients with rectal cancer. The CaPLR qualifies as a reliable inflammation-based predictor for survival outcome in rectal cancer patients after curative resection.

\section{Acknowledgments}

Funding: This work was supported by the National Basic Research Program of China (973 Program) (No. 2015CB554001), the National Natural Science Foundation of China (No. 81972245, YL; No. $81902877, \mathrm{HY}$ ), the Natural Science Fund for Distinguished Young Scholars of Guangdong Province (No. 2016A030306002, YL), the Tip-top Scientific and Technical Innovative Youth Talents of Guangdong special support program (No. 2015TQ01R454, YL), Sun Yat-sen University Clinical Research 5010 Program (No. 2018026, YL), the Natural Science Foundation of Guangdong Province (No. 2018A0303130303, HY), the "Five Five" Talent Team Construction Project of the Sixth Affiliated Hospital of Sun Yat-Sen University (No. P20150227202010244; No. P20150227202010251, YL), the Program of Introducing Talents of Discipline to Universities, and National Key Clinical Discipline [2012].

\section{Footnote}

Reporting Checklist: The authors have completed the TRIPOD reporting checklist. Available at http://dx.doi. 
org/10.21037/jgo-20-479

Data Sharing Statement: Available at http://dx.doi. org/10.21037/jgo-20-479

Peer Review File: Available at http://dx.doi.org/10.21037/ jgo-20-479

Conflicts of Interest: All authors have completed the ICMJE uniform disclosure form (available at http://dx.doi. org/10.21037/jgo-20-479). The authors have no conflicts of interest to declare.

Ethical Statement: The authors are accountable for all aspects of the work in ensuring that questions related to the accuracy or integrity of any part of the work are appropriately investigated and resolved. The study was conducted in accordance with the Declaration of Helsinki (as revised in 2013). This study was approved by the Institutional Review Board of the Sixth Affiliated Hospital of Sun Yat-sen University (2017ZSLYEC-006) and informed consent was taken from all the patients.

Open Access Statement: This is an Open Access article distributed in accordance with the Creative Commons Attribution-NonCommercial-NoDerivs 4.0 International License (CC BY-NC-ND 4.0), which permits the noncommercial replication and distribution of the article with the strict proviso that no changes or edits are made and the original work is properly cited (including links to both the formal publication through the relevant DOI and the license). See: https://creativecommons.org/licenses/by-nc-nd/4.0/.

\section{References}

1. Siegel RL, Miller KD, Jemal A. Cancer statistics, 2020. CA Cancer J Clin 2020;70:7-30.

2. Siegel RL, Miller KD, Goding SA, et al. Colorectal cancer statistics, 2020. CA Cancer J Clin 2020;70:145-64.

3. Watanabe T, Muro K, Ajioka Y, et al. Japanese Society for Cancer of the Colon and Rectum (JSCCR) guidelines 2016 for the treatment of colorectal cancer. Int J Clin Oncol 2018;23:1-34.

4. Horowitz M, Neeman E, Sharon E, et al. Exploiting the critical perioperative period to improve long-term cancer outcomes. Nat Rev Clin Oncol 2015;12:213-26.

5. Chen Z, Zhang P, Xu Y, et al. Surgical stress and cancer progression: the twisted tango. Mol Cancer 2019;18:132.

6. Tuomisto AE, Makinen MJ, Vayrynen JP. Systemic inflammation in colorectal cancer: Underlying factors, effects, and prognostic significance. World J Gastroenterol 2019;25:4383-404.

7. Greten FR, Grivennikov SI. Inflammation and Cancer: Triggers, Mechanisms, and Consequences. Immunity 2019;51:27-41.

8. Feliciano EMC, Kroenke CH, Meyerhardt JA, et al. Association of Systemic Inflammation and Sarcopenia With Survival in Nonmetastatic Colorectal Cancer: Results From the C SCANS Study. JAMA Oncol 2017;3:e172319.

9. Huang Z, Wang X, Zou Q, et al. High platelet-tolymphocyte ratio predicts improved survival outcome for perioperative NSAID use in patients with rectal cancer. Int J Colorectal Dis 2020;35:695-704.

10. Huang XZ, Chen WJ, Zhang X, et al. An Elevated Platelet-to-Lymphocyte Ratio Predicts Poor Prognosis and Clinicopathological Characteristics in Patients with Colorectal Cancer: A Meta-Analysis. Dis Markers 2017;2017:1053125.

11. Rossi S, Basso M, Strippoli A, et al. Are Markers of Systemic Inflammation Good Prognostic Indicators in Colorectal Cancer. Clin Colorectal Cancer 2017;16:264-74.

12. Bujak JK, Kosmala D, Szopa IM, et al. Inflammation, Cancer and Immunity-Implication of TRPV1 Channel. Front Oncol 2019;9:1087.

13. Iamartino L, Elajnaf T, Kallay E, et al. Calcium-sensing receptor in colorectal inflammation and cancer: Current insights and future perspectives. World J Gastroenterol 2018;24:4119-31.

14. Anderson KJ, Cormier RT, Scott PM. Role of ion channels in gastrointestinal cancer. World J Gastroenterol 2019;25:5732-72.

15. Shridhar K, Kinra S, Gupta R, et al. Serum Calcium Concentrations, Chronic Inflammation and Glucose Metabolism: A Cross-Sectional Analysis in the Andhra Pradesh Children and Parents Study (APCaPS). Curr Dev Nutr 2018;3:nzy085.

16. Lee YS, Nam HS, Lim JH, et al. Prognostic impact of a new score using neutrophil-to-lymphocyte ratios in the serum and malignant pleural effusion in lung cancer patients. BMC Cancer 2017;17:557.

17. Proctor MJ, Talwar D, Balmar SM, et al. The relationship between the presence and site of cancer, an inflammationbased prognostic score and biochemical parameters. Initial results of the Glasgow Inflammation Outcome Study. Br J Cancer 2010;103:870-6.

18. Huss L, Butt S, Borgquist S, et al. Serum levels of vitamin $\mathrm{D}$, parathyroid hormone and calcium in relation to 
survival following breast cancer. Cancer Causes Control 2014;25:1131-40.

19. Schwartz GG, Skinner HG. A prospective study of total and ionized serum calcium and time to fatal prostate cancer. Cancer Epidemiol Biomarkers Prev 2012;21:1768-73.

20. Proctor MJ, Morrison DS, Talwar D, et al. An inflammation-based prognostic score (mGPS) predicts cancer survival independent of tumour site: a Glasgow Inflammation Outcome Study. Br J Cancer 2011;104:726-34.

21. Huang Q, Qin H, Xiao J, et al. Association of tumor differentiation and prognosis in patients with rectal cancer undergoing neoadjuvant chemoradiation therapy. Gastroenterol Rep (Oxf) 2019;7:283-90.

22. Deng Y, Chi P, Lan P, et al. Modified FOLFOX6 With or Without Radiation Versus Fluorouracil and Leucovorin With Radiation in Neoadjuvant Treatment of Locally Advanced Rectal Cancer: Initial Results of the Chinese FOWARC Multicenter, Open-Label, Randomized Three-Arm Phase III Trial. J Clin Oncol 2016;34:3300-7.

23. Wittekind C. 2010 TNM system: on the 7 th edition of TNM classification of malignant tumors. Der Pathologe 2010;31:331-2.

24. Dickerson RN, Alexander KH, Minard G, et al. Accuracy of methods to estimate ionized and "corrected" serum calcium concentrations in critically ill multiple trauma patients receiving specialized nutrition support. JPEN J Parenter Enteral Nutr 2004;28:133-41.

25. Templeton AJ, Ace O, McNamara MG, et al. Prognostic role of platelet to lymphocyte ratio in solid tumors: a systematic review and meta-analysis. Cancer Epidemiol Biomarkers Prev 2014;23:1204-12.

26. Asano Y, Kashiwagi S, Onoda N, et al. Predictive Value of Neutrophil/Lymphocyte Ratio for Efficacy of Preoperative Chemotherapy in Triple-Negative Breast Cancer. Ann Surg Oncol 2016;23:1104-10.

27. Cuello-López J, Fidalgo-Zapata A, LópezAgudelo L, et al. Platelet-to-lymphocyte ratio as a predictive factor of complete pathologic response to neoadjuvant chemotherapy in breast cancer. PLoS One 2018;13:e0207224.

28. Ren DL, Li J, Yu HC, et al. Nomograms for predicting pathological response to neoadjuvant treatments in patients with rectal cancer. World J Gastroenterol 2019;25:118-37.

29. Deng Y, Chi P, Lan P, et al. Neoadjuvant Modified
FOLFOX6 With or Without Radiation Versus Fluorouracil Plus Radiation for Locally Advanced Rectal Cancer: Final Results of the Chinese FOWARC Trial. J Clin Oncol 2019;37:3223-33.

30. Shinto E, Hida JI, Ike H, et al. A New N Staging System for Colorectal Cancer in the Era of Extended Lymphadenectomy. Ann Surg Oncol 2018;25:3891-7.

31. Ozawa H, Nakanishi H, Sakamoto J, et al. Prognostic impact of the number of lateral pelvic lymph node metastases on rectal cancer. Jpn J Clin Oncol 2020;50:1254-60.

32. Haemmerle M, Stone RL, Menter DG, et al. The Platelet Lifeline to Cancer: Challenges and Opportunities. Cancer Cell 2018;33:965-83.

33. Rodland $\mathrm{KD}$. The role of the calcium-sensing receptor in cancer. Cell Calcium 2004;35:291-5.

34. Rand MD, Grimm LM, Artavanis-Tsakonas S, et al. Calcium depletion dissociates and activates heterodimeric notch receptors. Mol Cell Biol 2000;20:1825-35.

35. Wang W, Yu S, Huang S, et al. A Complex Role for Calcium Signaling in Colorectal Cancer Development and Progression. Mol Cancer Res 2019;17:2145-53.

36. Chen H, Liu Y, Jiang CJ, et al. Calcium-Activated Chloride Channel A4 (CLCA4) Plays Inhibitory Roles in Invasion and Migration Through Suppressing Epithelial-Mesenchymal Transition via PI3K/AKT Signaling in Colorectal Cancer. Med Sci Monit 2019;25:4176-85.

37. Liu S, Barry EL, Baron JA, et al. Effects of supplemental calcium and vitamin $\mathrm{D}$ on the APC/ $\beta$-catenin pathway in the normal colorectal mucosa of colorectal adenoma patients. Mol Carcinog 2017;56:412-24.

38. Tastanova A, Folcher M, Müller M, et al. Synthetic biology-based cellular biomedical tattoo for detection of hypercalcemia associated with cancer. Sci Transl Med 2018;10:eaap8562.

39. Martin TJ, Mundy GR. Bone metastasis: can osteoclasts be excluded. Nature 2007;445:E19; discussion E19-20.

40. Vassilopoulou-Sellin R, Newman BM, Taylor SH, et al. Incidence of hypercalcemia in patients with malignancy referred to a comprehensive cancer center. Cancer 1993;71:1309-12.

Cite this article as: Zhuang Z, Wang X, Huang M, Luo Y, $\mathrm{Yu}$ H. Serum calcium improved systemic inflammation marker for predicting survival outcome in rectal cancer. J Gastrointest Oncol 2021;12(2):568-579. doi: 10.21037/jgo-20-479 